\title{
Cores of hyperbolic 3-manifolds and limits of Kleinian groups II
}

\author{
James W. Anderson and Richard D. Canary* \\ Faculty of Mathematical Studies, University of Southampton \\ Highfield, Southampton, SO17 1BJ, England \\ Department of Mathematics, University of Michigan, Ann Arbor, MI 48109
}

\section{Introduction}

Troels Jörgensen conjectured that the algebraic and geometric limits of an algebraically convergent sequence of isomorphic Kleinian groups agree if there are no new parabolics in the algebraic limit. We prove that this conjecture holds in "most" cases. In particular, we show that it holds when the domain of discontinuity of the algebraic limit of such a sequence is non-empty (see Theorem 3.1). We further show, with the same assumptions, that the limit sets of the groups in the sequence converge to the limit set of the algebraic limit. As a corollary, we verify the conjecture for finitely generated Kleinian groups which are not (non-trivial) free products of surface groups and infinite cyclic groups (see Corollary 3.3). These results are extensions of similar results for purely loxodromic groups which can be found in [4]. Thurston [32] previously established these results in the case that the Kleinian groups are freely indecomposable (see also Ohshika [24], [25], [27]). Using different techniques than ours, Ohshika [26] has proven versions of these results for purely loxodromic function groups.

Both authors would like to thank the Institut Henri Poincaré for its hospitality during the writing of this paper, as well as the referee for useful comments.

\section{Preliminaries}

The purpose of this section is to describe the background material used in this paper.

${ }^{*}$ Research supported in part by the National Science Foundation and a fellowship from the Sloan Foundation 


\subsection{Convergence of Kleinian groups}

A Kleinian group is a discrete subgroup of $\mathrm{PSL}_{2}(\mathbf{C})$, which we view as acting either on hyperbolic 3 -space $\mathbf{H}^{3}$ via isometries or on the Riemann sphere $\overline{\mathbf{C}}$ via Möbius transformations. The action of $\Gamma$ partitions $\overline{\mathbf{C}}$ into the domain of discontinuity $\Omega(\Gamma)$, which is the largest open subset of $\overline{\mathbf{C}}$ on which $\Gamma$ acts properly discontinuously, and the limit set $\Lambda(\Gamma)$. A Kleinian group is non-elementary if its limit set contains at least three points, and is elementary otherwise. A Kleinian group is elementary if and only if it is virtually abelian; recall that a group is virtually abelian if it contains a finite index abelian subgroup. We refer the reader to Maskit [22] for a more detailed discussion of the theory of Kleinian groups.

It is often convenient to view a Kleinian group as the image of a discrete, faithful representation of a group into $\mathrm{PSL}_{2}(\mathbf{C})$. Given a finitely generated group $G$, let $\mathcal{D}(G)$ denote the space of all discrete, faithful representations of $G$ into $\operatorname{PSL}_{2}(\mathbf{C})$. A sequence $\left\{\rho_{j}\right\}$ in $\mathcal{D}(G)$ converges algebraically to $\rho$ if $\left\{\rho_{j}(g)\right\}$ converges to $\rho(g)$ for each $g \in G$. It is a fundamental result of Jørgensen [14] that $\mathcal{D}(G)$ is a closed subset of $\operatorname{Hom}\left(G, \mathrm{PSL}_{2}(\mathbf{C})\right)$ when $G$ is finitely generated and not virtually abelian.

An isomorphism $\alpha: \Phi \rightarrow \Theta$ between Kleinian groups $\Phi$ and $\Theta$ is type-preserving if $\varphi$ is parabolic if and only if $\alpha(\varphi)$ is parabolic for all $\varphi \in \Phi$. More generally, an algebraically convergent sequence $\left\{\rho_{j}\right\} \subset \mathcal{D}(G)$ with limit $\rho \in \mathcal{D}(G)$ is type-preserving if $\rho_{j} \circ \rho^{-1}: \rho(G) \rightarrow \rho_{j}(G)$ is type-preserving for all $j$.

A sequence $\left\{\Gamma_{j}\right\}$ of Kleinian groups converges geometrically to a Kleinian group $\widehat{\Gamma}$ if every element of $\widehat{\Gamma}$ is the limit of a sequence $\left\{\gamma_{j} \in \Gamma_{j}\right\}$ and if every accumulation point of every sequence $\left\{\gamma_{j} \in \Gamma_{j}\right\}$ lies in $\widehat{\Gamma}$. We make use of the following proposition, which assures that algebraically convergent sequences in $\mathcal{D}(G)$ have geometrically convergent subsequences.

Proposition 2.1 (Proposition 3.8 in Jørgensen and Marden [15]) If $G$ is not virtually abelian and $\left\{\rho_{j}\right\} \subset \mathcal{D}(G)$ is an algebraically convergent sequence with limit $\rho$, then there exists a subsequence $\left\{\rho_{j_{k}}\right\}$ of $\left\{\rho_{j}\right\}$ so that $\left\{\rho_{j_{k}}(G)\right\}$ converges geometrically to a Kleinian group $\widehat{\Gamma}$ with $\rho(G) \subset \widehat{\Gamma}$.

A sequence of closed sets $\left\{X_{j}\right\}$ in $\overline{\mathbf{C}}$ converges in the Hausdorff topology to a closed set $X$ in $\overline{\mathbf{C}}$ if every point of $X$ is the limit of a sequence of points $\left\{x_{j} \in X_{j}\right\}$ and if every accumulation point of a sequence $\left\{x_{j} \in X_{j}\right\}$ is contained in $X$. With this topology, the collection of non-empty closed subsets of $\overline{\mathbf{C}}$ is compact. Convergence of closed sets is always assumed to be in the Hausdorff topology.

If $\left\{\rho_{j}\right\} \subset \mathcal{D}$ converges algebraically to $\rho$ and $\left\{\rho_{j}(G)\right\}$ converges geometrically to $\rho(G)$, we say that $\left\{\rho_{j}\right\}$ converges strongly to $\rho$. It is conjectured that if $G$ is not virtually abelian and if $\left\{\rho_{j}\right\} \subset \mathcal{D}(G)$ converges algebraically to $\rho$, then $\left\{\Lambda\left(\rho_{j}(G)\right)\right\}$ converges to $\Lambda(\rho(G))$ if and only if 
$\left\{\rho_{j}\right\}$ converges strongly to $\rho$. We make use of the following partial result in the direction of this conjecture.

Proposition 2.2 (Proposition 4.2 in Jørgensen and Marden [15]) Let $G$ be a finitely generated group which is not virtually abelian. If $\left\{\rho_{j}\right\} \subset \mathcal{D}(G)$ converges algebraically to $\rho \in \mathcal{D}(G), \Omega(\rho(G))$ is non-empty, and $\left\{\Lambda\left(\rho_{j}(G)\right)\right\}$ converges to $\Lambda(\rho(G))$, then $\left\{\rho_{j}\right\}$ converges strongly to $\rho$.

The following lemma indicates the geometric significance, on the level of the quotient manifolds, of the geometric convergence of a sequence of Kleinian groups. (For a proof, see Theorem 3.2.9 of Canary, Epstein, and Green [10], and Theorem E.1.13 and Remark E.1.19 of Benedetti and Petronio [6].) Let 0 denote a fixed choice of basepoint for $\mathbf{H}^{3}$, and let $B_{R}(0)$ denote the ball of radius $R$ centered at 0 .

Lemma 2.3 A sequence of torsion-free Kleinian groups $\left\{\Gamma_{j}\right\}$ converges geometrically to a torsionfree Kleinian group $\widehat{\Gamma}$ if and only if there exists a sequence $\left\{\left(R_{j}, K_{j}\right)\right\}$ and a sequence of maps $\widetilde{f}_{j}: B_{R_{j}}(0) \rightarrow \mathbf{H}^{3}$ such that

1. $R_{j} \rightarrow \infty$ and $K_{j} \rightarrow 1$ as $j \rightarrow \infty$;

2. the map $\widetilde{f}_{j}$ is a $K_{j}$-bilipschitz diffeomorphism onto its image, $\widetilde{f}_{j}(0)=0$, and for any compact subset $A$ of $\mathbf{H}^{3},\left\{\left.\widetilde{f}_{j}\right|_{A}\right\}$ converges to the identity;

3. if $V_{j}=B_{R_{j}}(0) / \Gamma_{j}$, then $V_{j}$ is a submanifold of $N_{j}=\mathbf{H}^{3} / \Gamma_{j}$ and $\tilde{f}_{j}$ descends to a map $f_{j}: V_{j} \rightarrow \widehat{N}$, where $\widehat{N}=\mathbf{H}^{3} / \widehat{\Gamma}$; moreover, $f_{j}$ is also a $K_{j}$-bilipschitz diffeomorphism onto its image.

\subsection{Types of Kleinian groups}

Given a set $X \subset \mathbf{H}^{3} \cup \overline{\mathbf{C}}$ and a Kleinian group $\Gamma$, define the stabilizer of $X$ in $\Gamma$ to be

$$
\operatorname{st}_{\Gamma}(X)=\{\gamma \in \Gamma: \gamma(X)=X\}
$$

A component subgroup of $\Gamma$ is the stabilizer in $\Gamma$ of a component $\Delta$ of $\Omega(\Gamma)$. A set $X \subset \mathbf{H}^{3} \cup \overline{\mathbf{C}}$ is precisely invariant under a subgroup $\Phi$ of $\Gamma$ if $\operatorname{st}_{\Gamma}(X)=\Phi$ and if $X \cap \gamma(X)$ is empty for all $\gamma \in \Gamma-\Phi$.

There are several classes of Kleinian groups of particular interest in our paper. A quasifuchsian group is a finitely generated Kleinian group whose limit set is a Jordan curve and which contains no element interchanging the components of its domain of discontinuity, while an extended quasifuchsian group is a finitely generated Kleinian group whose limit set is a Jordan curve and which 
does contain an element interchanging the components of its domain of discontinuity. Note that an extended quasifuchsian group contains a canonical quasifuchsian subgroup of index two, namely the component subgroup associated to either of the components of its domain of discontinuity.

A degenerate group is a finitely generated Kleinian group whose domain of discontinuity and limit set are both non-empty and connected. A web group is a finitely generated Kleinian group whose domain of discontinuity contains infinitely many components, and each component subgroup is quasifuchsian. A finitely generated Kleinian group is a generalized web group if it is either quasifuchsian, extended quasifuchsian or a web group.

The convex core $C(N)$ of a hyperbolic 3-manifold $N=\mathbf{H}^{3} / \Gamma$ is the quotient of the convex hull $C H(\Lambda(\Gamma)$ ) of the limit set of $\Gamma$ by $\Gamma$. (The convex core can also be defined to be the smallest convex subset of $N$ whose inclusion is a homotopy equivalence.) A finitely generated Kleinian group is geometrically finite if its convex core has finite volume. A torsion-free Kleinian group is topologically tame if its quotient 3-manifold $N=\mathbf{H}^{3} / \Gamma$ is homeomorphic to the interior of a compact 3 -manifold. We note that geometrically finite Kleinian groups are topologically tame (see Marden [18]). A theorem of Scott [29] guarantees that any hyperbolic 3-manifold with finitely generated fundamental group contains a compact submanifold, called a compact core, whose inclusion is a homotopy equivalence. It is conjectured (see Marden [18]) that every hyperbolic 3-manifold with finitely generated fundamental group is homeomorphic to the interior of its compact core, and hence topologically tame.

\subsection{Decompositions of Kleinian groups}

In Section 3, we make use of two related decompositions, due to Abikoff and Maskit [1], of a finitely generated, torsion-free Kleinian group. We first discuss how a non-elementary, finitely generated, torsion-free Kleinian group with connected limit set and non-empty domain of discontinuity can be built from generalized web groups and degenerate groups without accidental parabolic elements. We then discuss the decomposition of a finitely generated Kleinian group into groups with connected limit sets and elementary groups.

We begin with a few definitions. Let $\Gamma$ be a torsion-free Kleinian group, and let $\gamma \in \Gamma$ be a parabolic element. A cusp region for $\gamma$ is a closed Jordan domain $D$ which is precisely invariant under $\langle\gamma\rangle$ in $\Gamma$ and intersects $\Lambda(\Gamma)$ only at the fixed point of $\gamma$. Note that this immediately implies that $\gamma$ is primitive in $\Gamma$ and that $\gamma$ cannot lie in a rank two abelian subgroup of $\Gamma$. The interior of a cusp region descends to a punctured disc neighborhood of a cusp on the Riemann surface $\Omega(\Gamma) / \Gamma$.

As the choice of a cusp region is by no means canonical, we introduce a notion of equivalence. Say that cusp regions $D$ and $D^{\prime}$ for parabolic elements $\gamma$ and $\gamma^{\prime}$ of $\Gamma$ are equivalent if their images on $\Omega(\Gamma) / \Gamma$ are neighborhoods of the same cusp on $\Omega(\Gamma) / \Gamma$. This implies in particular that $\gamma$ and $\gamma^{\prime}$ 
are conjugate in $\Gamma$; we note that the converse need not hold, as a single primitive parabolic element may stabilize two inequivalent cusp regions.

Let $\Gamma$ be a non-elementary, finitely generated, torsion-free Kleinian group with connected limit set; in particular, every component of $\Omega(\Gamma)$ is simply connected. Let $\Delta$ be a component of $\Omega(\Gamma)$, and let $\Phi=\operatorname{st}_{\Gamma}(\Delta)$ be its component subgroup. By the classical Uniformization Theorem for Riemann surfaces, there exists a conformal homeomorphism $f: \Delta \rightarrow \mathbf{H}^{2}$. The elements of $f \Phi f^{-1}$ are conformal homeomorphisms of $\mathbf{H}^{2}$, and so are necessarily Möbius transformations. An accidental parabolic element $\theta$ of $\Phi$ is a parabolic element whose conjugate $f \theta f^{-1}$ by $f$ is a primitive hyperbolic element of $f \Phi f^{-1}$. Denoting by $l$ the line in $\mathbf{H}^{2}$ joining the fixed points of $f \theta f^{-1}$, we define the axis of $\theta$ to be $c_{\theta}=f^{-1}(l)$ and the full axis of $\theta$ to be the Jordan curve $C_{\theta}=c_{\theta} \cup \operatorname{fix}(\theta)$; for a detailed discussion of accidental parabolics, we refer the reader to Maskit [22], particularly Chapter IX.D.10. By construction, $c_{\theta}$ is precisely invariant under $\Theta=\langle\theta\rangle$ in $\Gamma$, and $C_{\theta}$ separates $\Lambda(\Phi)$, and hence separates $\Lambda(\Gamma)$.

Before describing the decomposition of a Kleinian group along an accidental parabolic element, we make a couple of remarks. First, a given parabolic element of $\Gamma$ may lie in several component subgroups, being accidental in some and not accidental in others; hence, we make the convention that whenever we refer to an accidental parabolic element of $\Gamma$, we actually refer to the parabolic element along with the implicit choice for the component of $\Omega(\Gamma)$ containing its axis. Second, a primitive parabolic element of a Kleinian group which keeps invariant a component of the domain of discontinuity must either be accidental in that component or must have a cusp region in that component. Third, a finitely generated, non-elementary Kleinian group with connected limit set and non-empty domain of discontinuity contains no accidental parabolic elements if and only if it is either a degenerate group without accidental parabolics or a generalized web group (this follows immediately from Theorem 4 of Maskit [20]).

Given an accidental parabolic element $\theta$ of $\Gamma$ with full axis $C_{\theta}$, let $P_{1}$ and $P_{2}$ be the two components of $\overline{\mathbf{C}}-\Gamma\left(C_{\theta}\right)$ which contain $C_{\theta}$ in their boundaries, and let $\Gamma_{m}$ be the stabilizer of $P_{m}$ in $\Gamma$. Let $E_{1}$ and $E_{2}$ be the closed discs in $\overline{\mathbf{C}}$ determined by $C_{\theta}$, labeled so that $C_{\theta}$ separates the interior of $E_{m}$ from $P_{m}$.

Suppose that $P_{1}$ and $P_{2}$ are not equivalent under $\Gamma$. In this case, we say that $C_{\theta}$ is a separating full axis for $\theta$, and we refer to $\Gamma_{1}$ and $\Gamma_{2}$ as the factor subgroups of this decomposition. It is easy to show that $\Gamma$ is generated by its factor subgroups $\Gamma_{1}$ and $\Gamma_{2}$, that both factor subgroups are nonelementary and finitely generated, that $\Gamma_{1} \cap \Gamma_{2}=\langle\theta\rangle$, and that $C_{\theta}$ is precisely invariant under $\langle\theta\rangle$ in $\Gamma_{m}$ for both $m$. The statement of the Klein-Maskit combination theorem of type I given below, adapted from the statement in Maskit [22], essentially states that this operation of decomposition can be reversed. 
Recall that a fundamental domain for a finitely generated Kleinian group $\Gamma$ is an open subset $D$ of $\Omega(\Gamma)$ so that $D$ is precisely invariant under the identity, every point of $\Omega(\Gamma)$ is equivalent under the action of $\Gamma$ to a point of $\bar{D}$ and the boundary of $D$ is a finite collection of analytic arcs.

Theorem 2.4 (Klein-Maskit combination I) Let $\Gamma_{1}$ and $\Gamma_{2}$ be non-elementary, finitely generated Kleinian groups whose intersection $\Gamma_{1} \cap \Gamma_{2}$ is the parabolic cyclic group $\langle\theta\rangle$. Let $C$ be a Jordan curve in $\overline{\mathbf{C}}$ determining closed discs $E_{1}$ and $E_{2}$, so that $E_{m}$ is a cusp region for $\theta$ in $\Gamma_{m}$.

Then, the following hold. First, the group $\Gamma=\left\langle\Gamma_{1}, \Gamma_{2}\right\rangle$ is a Kleinian group isomorphic to the amalgamated free product of $\Gamma_{1}$ and $\Gamma_{2}$ along $\langle\theta\rangle$. Second, if $D_{m}$ is a fundamental domain for $\Gamma_{m}$ so that $D_{m} \cap E_{m}$ is a fundamental domain for the action of $\langle\theta\rangle$ on $E_{m}, D_{m} \cap E_{3-m}$ has non-empty interior, and $D_{1} \cap C_{\theta}=D_{2} \cap C_{\theta}$, then $D=\left(D_{1} \cap E_{2}\right) \cup\left(D_{2} \cap E_{1}\right)$ is a fundamental domain for the action of $\Gamma$. Third, every cusp region for $\Gamma_{m}$ which does not intersect any $\Gamma_{m}$-translate of $E_{m}$ is a cusp region for $\Gamma$, and every cusp region for $\Gamma$ is a cusp region for either $\Gamma_{1}$ or $\Gamma_{2}$.

Suppose, on the other hand, that $P_{1}$ and $P_{2}$ are equivalent by $\xi \in \Gamma$, so that $\xi\left(P_{1}\right)=P_{2}$. In this case we say that $C_{\theta}$ is a non-separating full axis for $\theta$, and we refer to $\Gamma_{2}$ and $\langle\xi\rangle$ as the factor subgroups of this decomposition. It is easy to show that $\Gamma$ is generated by its factor subgroups $\Gamma_{2}$ and $\langle\xi\rangle$, that $\Gamma_{2}$ is finitely generated and non-elementary, that $\Gamma_{2} \cap \xi^{-1} \Gamma_{2} \xi=\langle\theta\rangle$, and that $C_{\theta}$ is precisely invariant under $\langle\theta\rangle$ in $\Gamma_{2}$ (even though we may have that $\xi\left(C_{\theta}\right) \cap C_{\theta}$ is non-empty if $\xi$ and $\theta$ commute). The statement of the Klein-Maskit combination theorem of type II, adapted from the statement in Maskit[22], essentially states that this decomposition can be reversed.

Theorem 2.5 (Klein-Maskit combination II) Let $\Gamma^{0}$ be a non-elementary, finitely generated Kleinian group, and let $\left\langle\theta_{1}\right\rangle$ and $\left\langle\theta_{2}\right\rangle$ be parabolic cyclic subgroups of $\Gamma^{0}$. Let $C_{1}$ and $C_{2}$ be Jordan curves in $\overline{\mathbf{C}}$ so that, for both $m, C_{m}$ determines a closed disc $E_{m}$ which is a cusp region for $\left\langle\theta_{m}\right\rangle$ in $\Gamma^{0}$. We also require that $E_{1}$ and $E_{2}$ are inequivalent cusp regions for $\Gamma^{0}$. Let $\xi$ be a Möbius transformation so that $\xi \theta_{1} \xi^{-1}=\theta_{2}, \xi\left(C_{1}\right)=C_{2}$, and the image of the interior of $E_{1}$ under $\xi$ is disjoint from the interior of $E_{2}$.

Then, the following hold. First, the group $\left\langle\Gamma^{0}, \xi\right\rangle$ is a Kleinian group isomorphic to the HNN extension of $\Gamma^{0}$ by $\xi$. Second, if $D^{0}$ is a fundamental domain for $\Gamma^{0}$ so that $D^{0} \cap E_{m}$ is a fundamental domain for the action of $\left\langle\theta_{m}\right\rangle$ on $E_{m}$ and $\xi\left(D_{1} \cap C_{1}\right)=D_{2} \cap C_{2}$, then $D=D^{0} \cap A$ is a fundamental domain for $\Gamma$, where $A=\overline{\mathbf{C}}-\left(E_{1} \cup E_{2}\right)$. Third, every cusp region for $\Gamma^{0}$ which does not intersect any $\Gamma^{0}$-translate of $E_{1}$ or $E_{2}$ is a cusp region for $\Gamma$, and every cusp region for $\Gamma$ is a cusp region for $\Gamma^{0}$.

Abikoff and Maskit [1] showed that given a finitely generated, non-elementary Kleinian group $\Gamma$ with connected limit set and non-empty domain of discontinuity, one can produce a finite collection 
$\left\{\Gamma_{1}, \ldots, \Gamma_{k}, \Gamma_{k+1}, \ldots, \Gamma_{l}\right\}$ of subgroups of $\Gamma$, where $\Gamma_{i}$ is either a generalized web group or a degenerate group without accidental parabolic elements for $i \leq k$, and $\Gamma_{i}$ is a cyclic group for $i>k$, so that $\Gamma$ can be built from $\left\{\Gamma_{1}, \ldots, \Gamma_{l}\right\}$ by repeatedly performing Klein-Maskit combinations of types I and II. Specifically, set $\Gamma^{1}=\Gamma_{1}$; for $j \leq k$, form $\Gamma^{j}$ from $\Gamma^{j-1}$ and $\Gamma_{j}$ by using a Klein-Maskit combination of type I along a common parabolic cyclic subgroup of $\Gamma^{j-1}$ and $\Gamma_{j}$, and for $j>k$, form $\Gamma^{j}$ from $\Gamma^{j-1}$ and $\Gamma_{j}$ by a Klein-Maskit combination of type II, where the generator of $\Gamma_{j}$ pairs inequivalent cusp regions of $\Gamma^{j-1}$. The final result of this process $\Gamma^{l}$ is the original group $\Gamma$.

Abikoff and Maskit [1] also showed that any torsion-free, finitely generated Kleinian group with non-empty domain of discontinuity can be constructed from a finite collection of elementary groups and groups with connected limit set by a finite number of applications of the Klein combination theorem. We recall the statement of the Klein combination theorem below for reference, and refer the reader to Maskit [22] for a complete discussion.

Theorem 2.6 (Klein combination theorem) Let $\Gamma_{1}$ and $\Gamma_{2}$ be Kleinian groups with non-empty domains of discontinuity, and suppose there exist fundamental domains $D_{1}$ and $D_{2}$ for $\Gamma_{1}$ and $\Gamma_{2}$ so that each contains the exterior of the other. Then, $\Gamma=\left\langle\Gamma_{1}, \Gamma_{2}\right\rangle$ is a Kleinian group isomorphic to the free product $\Gamma_{1} * \Gamma_{2}$, and $D=D_{1} \cap D_{2}$ is a fundamental domain for $\Gamma$.

\subsection{Relative compact cores and ends of Kleinian groups}

A horoball in $\mathbf{H}^{3}$ is an open Euclidean ball (or half-space) $B$ in $\mathbf{H}^{3}$ whose (Euclidean) closure in $\mathbf{H}^{3} \cup \overline{\mathbf{C}}$ intersects $\overline{\mathbf{C}}$ in a single point, which is the center of the horoball. A precisely invariant system of horoballs $\mathcal{H}$ for a Kleinian group $\Gamma$ is a $\Gamma$-invariant collection of disjoint horoballs centered at parabolic fixed points of $\Gamma$, such that there is one based at every parabolic fixed point. It is a consequence of the Margulis Lemma (see Benedetti and Petronio [6] or Maskit [22]) that every Kleinian group has a precisely invariant system of horoballs. Given a precisely invariant system of horoballs $\mathcal{H}$ for $\Gamma$, set $N_{\mathcal{H}}=\left(\mathbf{H}^{3}-\mathcal{H}\right) / \Gamma$.

A relative compact core of $N_{\mathcal{H}}$ is a compact submanifold $M$ of $N_{\mathcal{H}}$ so that the inclusion of $M$ into $N_{\mathcal{H}}$ is a homotopy equivalence and so that $\partial M$ intersects every non-compact component of $\partial N_{\mathcal{H}}$ in an incompressible annulus and contains every toroidal component of $\partial N_{\mathcal{H}}$. (Results of McCullough [23] or Kulkarni and Shalen [17] guarantee that $N_{\mathcal{H}}$ has a relative compact core whenever $\pi_{1}(N)$ is finitely generated.) Set $P=\partial M \cap \partial N_{\mathcal{H}}$. The ends of $N_{\mathcal{H}}$ are in one-to-one correspondence with the components of $\partial M-P$ (see Bonahon [7]). An end $E$ of $N_{\mathcal{H}}$ is geometrically finite if it has a neighborhood $U$ such that $U \cap C(N)=\emptyset$, and is geometrically infinite otherwise. We also refer to the corresponding components of $\partial M-P$ as geometrically finite or geometrically infinite.

The following generalization of Thurston's covering theorem [32] appears in [9]. 
Theorem 2.7 Let $N=\mathbf{H}^{3} / \Gamma$ be a topologically tame hyperbolic 3-manifold which covers another hyperbolic 3-manifold $\widehat{N}$ by a local isometry $\pi: N \rightarrow \widehat{N}$, and suppose that $\Omega(\Gamma)$ is non-empty. If $E$ is a geometrically infinite end of $N_{\mathcal{H}}$, then $E$ has a neighborhood $U$ such that $\pi$ is finite-to-one on $U$.

The relative compact core encodes much of the structure of the Kleinian group. For example, a finitely generated, torsion-free Kleinian group $\Gamma$ with associated relative compact core $(M, P)$ has connected limit set if and only if every geometrically finite component of $\partial M-P$ is incompressible. In this language, an accidental parabolic gives rise to an essential annulus $A$ in $M$ which has one boundary component in $P$ and the other in a geometrically finite component of $\partial M-P$. If $\Gamma$ has connected limit set, then the non-cyclic subgroups in the Abikoff-Maskit decomposition of $\Gamma$ are simply the fundamental groups of the components of $M-\mathcal{A}$, where $\mathcal{A}$ is a maximal collection of disjoint, non-parallel essential annuli associated to accidental parabolics. If $\Gamma$ has disconnected limit set, the non-cyclic subgroups in an Abikoff-Maskit decomposition of $\Gamma$ into groups with connected limit set and elementary groups arise as fundamental groups of components of $M-\mathcal{C}$, where $\mathcal{C}$ is a maximal collection of disjoint, non-parallel compressing disks for $(M, P)$ whose boundary curves lie in geometrically finite components of $\partial M-P$.

\section{The main results}

In this section we prove our main result, namely that a type-preserving sequence converges strongly, under the assumption that the domain of discontinuity of the algebraic limit is non-empty.

Theorem 3.1 Let $G$ be a finitely generated, torsion-free group, and let $\left\{\rho_{j}\right\} \subset \mathcal{D}(G)$ be a typepreserving sequence converging algebraically to $\rho \in \mathcal{D}(G)$. If $\Omega(\rho(G))$ is non-empty, then $\left\{\rho_{j}\right\}$ converges strongly to $\rho$ and $\left\{\Lambda\left(\rho_{j}(G)\right)\right\}$ converges to $\Lambda(\rho(G))$.

Before beginning the proof, we recall the statement of Theorem F from [4].

Theorem 3.2 (Theorem $F$ from [4]) Let $G$ be a finitely generated, torsion-free, non-abelian group and let $\left\{\rho_{j}\right\}$ be a sequence in $\mathcal{D}(G)$ converging to $\rho$. If $\Lambda(\rho(G))=\overline{\mathbf{C}}$ and $G$ is not a non-trivial free product of (orientable) surface groups and cyclic groups, then $\left\{\rho_{j}\right\}$ converges strongly to $\rho$. Moreover, $\left\{\Lambda\left(\rho_{j}(G)\right)\right\}$ converges to $\Lambda(\rho(G))=\overline{\mathbf{C}}$.

Combining Theorem 3.1 with the above theorem yields the following immediate corollary. 
Corollary 3.3 Let $G$ be a finitely generated, torsion-free group, and let $\left\{\rho_{j}\right\} \subset \mathcal{D}(G)$ be a typepreserving sequence converging algebraically to $\rho$. If $G$ is not a (non-trivial) free product of (orientable) surface groups and infinite cyclic groups, then $\left\{\rho_{j}\right\}$ converges strongly to $\rho$ and $\left\{\Lambda\left(\rho_{j}(G)\right)\right\}$ converges to $\Lambda(\rho(G))$.

We further note that we obtain the same conclusion if we replace the group-theoretic assumption in the statement of the corollary with the weaker topological assumption that the relative compact core of the algebraic limit is not a relative compression body. For a discussion of the relation between the group-theoretic and topological conditions, we refer the reader to Section 11 of [4].

Proof of Theorem 3.1: The proof divides naturally into three steps. In Step 1, we show that Theorem 3.1 holds in the case that $\rho(G)$ is either a generalized web group, a degenerate group without accidental parabolics or an elementary group. In Step 2, we show that Theorem 3.1 holds in the case that $\rho(G)$ has connected limit set, using the result of Step 1 and the Abikoff-Maskit decomposition of $\rho(G)$ into generalized web groups, degenerate groups without accidental parabolic elements and elementary groups. In Step 3, we pass from the case of connected limit set to the general case, making use of the Abikoff-Maskit decomposition of a torsion-free finitely generated Kleinian group into groups with connected limit sets and elementary groups.

Step 1: We recall that Thurston [32] and Kerckhoff and Thurston [16] established Theorem 3.1 in the case that the algebraic limit is a degenerate group without accidental parabolic elements. One can obtain this by combining Theorem 9.6.1 in Thurston [32] with the proof of Corollary 2.2 in Kerckhoff and Thurston [16]; see also Corollary 6.1 in Ohshika [27].

We next establish Theorem 3.1 for generalized web groups.

Proposition 3.4 Let $G$ be a finitely generated, torsion-free, non-abelian group, and let $\left\{\rho_{j}\right\} \subset$ $\mathcal{D}(G)$ be a type-preserving sequence converging algebraically to $\rho$. If $\rho(G)$ is a generalized web group, then $\left\{\rho_{j}\right\}$ converges strongly to $\rho$ and $\left\{\Lambda\left(\rho_{j}(G)\right)\right\}$ converges to $\Lambda(\rho(G))$.

Proof of Proposition 3.4: Let $\Gamma^{0}$ be a geometrically finite subgroup of $\rho(G)$. Since each $\rho_{j} \circ \rho^{-1}$ is type-preserving, Marden's quasiconformal stability theorem (Proposition 9.1 in Marden [18]) implies that $\Gamma_{j}^{0}=\rho_{j} \circ \rho^{-1}\left(\Gamma^{0}\right)$ is geometrically finite and quasiconformally conjugate to $\rho(G)$ for sufficiently large $j$. In particular, there exists a sequence $\left\{\phi_{j}\right\}$ of $k_{j}$-quasiconformal maps of $\overline{\mathbf{C}}$ converging to the identity map, with $\left\{k_{j}\right\}$ converging to 1 , so that $\rho_{j} \circ \rho^{-1}(\gamma)=\phi_{j} \circ \gamma \circ \phi_{j}^{-1}$ for all $\gamma \in \Gamma^{0}$. One sees immediately that $\left\{\Gamma_{j}^{0}\right\}$ converges geometrically to $\Gamma^{0}$ and that $\left\{\Lambda\left(\Gamma_{j}^{0}\right)\right\}$ converges to $\Lambda\left(\Gamma^{0}\right)$. Hence, Theorem 3.1 holds whenever $\rho(G)$ is geometrically finite, and in particular for $\rho(G)$ quasifuchsian or extended quasifuchsian. 
Suppose now that $\rho(G)$ is a web group. Proposition 2.2 implies that it suffices to prove that $\left\{\Lambda\left(\rho_{j}(G)\right\}\right.$ converges to $\Lambda(\rho(G))$. If $\left\{\Lambda\left(\rho_{j}(G)\right)\right\}$ does not converge to $\Lambda(\rho(G))$, then there exists a subsequence of $\left\{\rho_{j}\right\}$, again called $\left\{\rho_{j}\right\}$, so that $\left\{\Lambda\left(\rho_{j}(G)\right)\right\}$ converges to a set $\widehat{\Lambda}$ which contains $\Lambda(\rho(G))$ as a proper subset. In particular, there must exist a quasifuchsian component subgroup of $\rho(G)$ whose limit set separates $\widehat{\Lambda}$. Proposition 2.1 implies that we may pass to a further subsequence of $\left\{\rho_{j}\right\}$, still called $\left\{\rho_{j}\right\}$, such that $\left\{\rho_{j}(G)\right\}$ converges geometrically to a Kleinian group $\widehat{\Gamma}$. The definitions of geometric and Hausdorff convergence imply that $\Lambda(\rho(G)) \subset \Lambda(\widehat{\Gamma}) \subset \widehat{\Lambda}$.

Let $x \in \widehat{\Lambda}-\Lambda(\rho(G))$, let $\Delta$ be the component of $\Omega(\rho(G))$ which contains $x$, and set $\Phi=$ $\operatorname{st}_{\rho(G)}(\Delta)$, so that $\Lambda(\Phi)$ separates $\widehat{\Lambda}$. Set $\Phi_{j}=\rho_{j} \circ \rho^{-1}(\Phi)$. Since $\rho(G)$ is a web group, $\Phi$ is quasifuchsian, and hence geometrically finite. Let $\left\{\phi_{j}\right\}$ be the sequence of quasiconformal maps conjugating $\Phi_{j}$ to $\Phi$ produced in the first paragraph of this proof. Since $\Lambda(\Phi)$ separates $\widehat{\Lambda},\left\{\Lambda\left(\rho_{j}(G)\right)\right\}$ converges to $\widehat{\Lambda}$, and $\left\{\phi_{j}\right\}$ converges to the identity map, we see that $\Lambda\left(\Phi_{j}\right)=\phi_{j}(\Lambda(\Phi))$ separates $\Lambda\left(\rho_{j}(G)\right)$ for all sufficiently large $j$.

Recall that a spanning disc for the quasifuchsian subgroup $\Phi$ of the Kleinian group $\rho(G)$ is a properly embedded open disc $D$ in $\mathbf{H}^{3}$ which is precisely invariant under $\Phi$ in $\rho(G)$ and which extends to a closed disc $\bar{D}$ in $\mathbf{H}^{3} \cup \overline{\mathbf{C}}$ with boundary $\Lambda(\Phi)$. Let $N=\mathbf{H}^{3} / \rho(G)$ and $\widehat{N}=\mathbf{H}^{3} / \widehat{\Gamma}$, and $\pi: N \rightarrow \widehat{N}$ and $\widehat{p}: \mathbf{H}^{3} \rightarrow \widehat{N}$ be the associated covering maps. It is shown in the proof of Proposition 6.1 of [4] that, for any algebraically convergent sequence in $\mathcal{D}(G)$ whose algebraic limit is a web group, such as $\rho(G)$, and for any component subgroup of $\rho(G)$, such as $\Phi$, there exists a spanning disk $D$ for $\Phi$ and a compact core $M$ for $N$ such that $\pi$ is an embedding restricted to $M$, $\widehat{p}(D)$ is a properly embedded surface in $\widehat{N}$, and $\pi(M)$ is disjoint from $\widehat{p}(D)$.

Set $N_{j}=\mathbf{H}^{3} / \rho_{j}(G)$. Let $\left\{V_{j} \subset N_{j}\right\}$ and $\left\{f_{j}: V_{j} \rightarrow \widehat{N}\right\}$ be the sequence of submanifolds and $K_{j}$-bilipschitz diffeomorphisms produced by Lemma 2.3. Since $\pi(M)$ lies in $f_{j}\left(V_{j}\right)$ for all sufficiently large $j$, the submanifold $M_{j}=f_{j}^{-1}(\pi(M))$ of $N_{j}$ is defined. In Corollary C of [4], we observe that $M_{j}$ is a compact core for $N_{j}$ for all sufficiently large $j$.

One may extend each $\phi_{j}$ to a $L_{j}$-bilipschitz diffeomorphism $\psi_{j}: \mathbf{H}^{3} \rightarrow \mathbf{H}^{3}$ conjugating the action of $\Phi$ to the action of $\Phi_{j}$ (see Douady and Earle [11], Reimann [28], or Tukia [33]) in such a way that $\left\{L_{j}\right\}$ converges to 1 and $\left\{\psi_{j}\right\}$ converges to the identity map. Let $D_{j}=\psi_{j}(D)$, and note that $D_{j}$ is a properly embedded open disc in $\mathbf{H}^{3}$ which is invariant under $\Phi_{j}$ and which extends to a closed disc in $\mathbf{H}^{3} \cup \overline{\mathbf{C}}$ with boundary $\Lambda\left(\Phi_{j}\right)$. However, $D_{j}$ need not be a spanning disk for $\Phi_{j}$, as it need not be precisely invariant under $\Phi_{j}$ in $\rho_{j}(G)$.

We next show that $p_{j}\left(D_{j}\right)$ is disjoint from $M_{j}$ for sufficiently large $j$, where $p_{j}: \mathbf{H}^{3} \rightarrow N_{j}$ is the covering map. Choose $\delta>0$ so that $\min _{x \in \pi(M)} \operatorname{inj}_{\widehat{N}}(x)>3 \delta$. Since $f_{j}: V_{j} \rightarrow \widehat{N}$ is $K_{j}$-bilipschitz and $\left\{K_{j}\right\}$ converges to 1 , we see that $\min _{x \in M_{j}} \operatorname{inj}_{N_{j}}(x)>2 \delta$ for sufficiently large $j$. 
Let $X \subset D$ denote the set of points for which there exists a non-trivial $\gamma \in \Phi$ such that $\mathrm{d}(x, \gamma(x))<\delta$, and let $Y=D-X$. Consider the subsets $Y_{j}=\psi_{j}(Y)$ and $X_{j}=\psi_{j}(X)$ of $D_{j}$. For each $x \in X_{j}$, there exists a non-trivial $\gamma_{j} \in \Phi_{j}$ so that $\mathrm{d}\left(x, \gamma_{j}(x)\right)<L_{j} \delta$. Since $L_{j} \delta<$ $\min _{x \in M_{j}} \operatorname{inj}_{N_{j}}(x)$ for sufficiently large $j$, we have that $p_{j}\left(X_{j}\right)$ is disjoint from $M_{j}$. Moreover, since $\widehat{p}(Y)$ is compact and is disjoint from $\pi(M)$, and since both $\left\{f_{j}\right\}$ and $\left\{\psi_{j}\right\}$ converge to the identity map, $p_{j}\left(Y_{j}\right)$ is disjoint from $M_{j}$ for sufficiently large $j$. This completes the proof that $p_{j}\left(D_{j}\right)$ is disjoint from $M_{j}$ for sufficiently large $j$.

We now assume that we have chosen $j$ large enough so that $\Lambda\left(\Phi_{j}\right)$ separates $\Lambda\left(\rho_{j}(G)\right), M_{j}$ is a compact core for $N_{j}$, and $p_{j}\left(D_{j}\right)$ is disjoint from $M_{j}$. Since $\Lambda\left(\Phi_{j}\right)$ separates $\Lambda\left(\rho_{j}(G)\right)$ and since pairs of fixed points of loxodromic elements of $\rho_{j}(G)$ are dense in $\Lambda\left(\rho_{j}(G)\right) \times \Lambda\left(\rho_{j}(G)\right.$ ) (see Proposition V.E.5 in [22]), there exists a hyperbolic element $\gamma_{j} \in \rho_{j}(G)$ whose fixed points are separated by $\Lambda\left(\Phi_{j}\right)$. If $C_{j}$ is the closed geodesic in $N_{j}$ which is the projection of the axis of $\gamma_{j}$, then every curve homotopic to $C_{j}$ must intersect $p_{j}\left(D_{j}\right)$. However, since $M_{j}$ is a compact core for $N_{j}$ which is disjoint from $p_{j}\left(D_{j}\right)$, there exists a curve in $M_{j}$ which is homotopic to $C_{j}$ and disjoint from $p_{j}\left(D_{j}\right)$. This contradiction establishes the result.

Proposition 3.4

We note the following corollary of the proof of Proposition 3.4.

Corollary 3.5 Let $G$ be a finitely generated, torsion-free, non-abelian group, and let $\left\{\rho_{j}\right\} \subset \mathcal{D}(G)$ be a type-preserving sequence converging algebraically to $\rho$. Suppose that $\rho(G)$ is a generalized web group, and let $\Phi$ be a component subgroup of $\rho(G)$. Then, $\Phi_{j}=\rho_{j} \circ \rho^{-1}(\Phi)$ is quasifuchsian and is a component subgroup of $\rho_{j}(G)$ for all sufficiently large $j$.

We complete Step 1 by establishing Theorem 3.1 for elementary groups, under the additional assumption that the limit representation $\rho$ is discrete and faithful.

Lemma 3.6 Let $G$ be a free abelian group of rank at most two, and let $\left\{\rho_{j}\right\}$ be a type-preserving sequence in $\mathcal{D}(G)$ converging algebraically to $\rho \in \mathcal{D}(G)$. Then, $\left\{\rho_{j}\right\}$ converges strongly to $\rho$.

Proof of Lemma 3.6: Suppose there exists a sequence of non-trivial elements $\left\{g_{j}\right\}$ of $G$ so that $\left\{\rho_{j}\left(g_{j}\right)\right\}$ converges to a Möbius transformation $\gamma$. We wish to show that $\gamma \in \rho(G)$. We begin by noting that $\operatorname{fix}(\gamma)=\Lambda(\rho(G))$, for otherwise, the fixed point set fix $\left(\rho_{j}\left(g_{j}\right)\right)$ of $\rho_{j}\left(g_{j}\right)$ would be distinct from $\Lambda\left(\rho_{j}(G)\right)$ for all sufficiently large $j$, which cannot occur.

Suppose that $G=\langle g\rangle$ is infinite cyclic and that $\rho(g)$ is loxodromic. As $G$ is cyclic, we may write $g_{j}=g^{n_{j}}$ for $n_{j} \in \mathbf{Z}$. Since $\left\{\rho_{j}(g)\right\}$ converges to $\rho(g)$, and since $\rho_{j}(g)$ is loxodromic for all $j$ and $\rho(g)$ 
is loxodromic, there exists a sequence of Möbius transformations $\left\{\beta_{j}\right\}$ converging to the identity so that the axis of $\beta_{j} \rho_{j}(g) \beta_{j}^{-1}$ in $\mathbf{H}^{3}$ is equal to the axis $l$ of $\rho(g)$ in $\mathbf{H}^{3}$ for all $j$. Since $\left\{\beta_{j}\right\}$ converges to the identity, $\left\{\beta_{j} \rho_{j}\left(g_{j}\right) \beta_{j}^{-1}\right\}$ converges to $\gamma$, and so the translation length of $\beta_{j} \rho_{j}\left(g_{j}\right) \beta_{j}^{-1}$ along $l$ converges to the translation length of $\gamma$.

If $\left\{n_{j}\right\}$ is not bounded, there exists a subsequence of $\left\{n_{j}\right\}$, again called $\left\{n_{j}\right\}$, converging to either $\infty$ or $-\infty$. Since the translation length of $\rho_{j}\left(g_{j}\right)$ is $n_{j}$ times the translation length of $\beta_{j} \rho_{j}(g) \beta_{j}^{-1}$, we see that the translation length of $\beta_{j} \rho_{j}(g) \beta_{j}^{-1}$ along $l$ converges to zero, and so $\rho(g)$ fixes $l$ pointwise. In particular, $\rho(g)$ is either the identity or elliptic. However, $\rho(g)$ cannot be the identity, as this would contradict the faithfulness of $\rho$. Also, $\rho(g)$ cannot be elliptic, as this would contradict either the faithfulness of $\rho$ if $\rho(g)$ has finite order or the discreteness of $\rho(G)$ if $\rho(g)$ has infinite order. This contradiction gives that $\left\{n_{j}\right\}$ is bounded. If $\left\{n_{j}\right\}$ is not eventually constant, there exist two subsequences with limits $m \neq n$, and so there are two subsequences of $\left\{\rho_{j}\left(g_{j}\right)\right\}$ whose limits are $\rho(g)^{m}$ and $\rho(g)^{n}$, which cannot occur. Hence, $\left\{n_{j}\right\}$ is eventually constant, and so $\gamma \in \rho(G)$.

The proof in the case that $\rho(G)$ is a parabolic subgroup is similar. Suppose that $G$ is infinite cyclic and $\rho(g)$ is parabolic. Since $\left\{\rho_{j}(g)\right\}$ converges to $\rho(g)$, and since $\rho_{j}(g)$ is parabolic for all $j$ and $\rho(g)$ is parabolic, there exists a sequence of Möbius transformations $\left\{\beta_{j}\right\}$ converging to the identity so that $\beta_{j} \rho_{j}(g) \beta_{j}^{-1}=\rho(g)$ for all $j$. Normalizing so that $\rho(g)(z)=z+1$ and writing $g_{j}=g^{n_{j}}$ for $n_{j} \in \mathbf{Z}$, we see that $\beta_{j} \rho_{j}\left(g_{j}\right) \beta_{j}^{-1}(z)=z+n_{j}$. Since $\left\{\beta_{j} \rho_{j}\left(g_{j}\right) \beta_{j}^{-1}\right\}$ converges to a Möbius transformation $\gamma$, it is easy to see that $\left\{n_{j}\right\}$ must be eventually constant, and so $\gamma \in \rho(G)$.

In the case that $G$ has rank two, choose generators $a$ and $b$. Since $\rho_{j}(a)$ and $\rho_{j}(b)$ are parabolic for all $j$, and since $\rho(a)$ and $\rho(b)$ are parabolic, there exists a sequence of Möbius transformations $\left\{\beta_{j}\right\}$ converging to the identity so that $\beta_{j} \rho_{j}(a) \beta_{j}^{-1}=\rho(a)$ for all $j$. Normalizing so that $\rho(a)(z)=$ $z+1$, we see that $\beta_{j} \rho_{j}(b) \beta_{j}^{-1}(z)=z+\tau_{j}$ and $\rho(b)(z)=z+\tau$, where $\left\{\tau_{j}\right\}$ converges to $\tau$ and $\tau$ has non-zero imaginary part. Write $g_{j}=a^{n_{j}} b^{m_{j}}$ for $n_{j}, m_{j} \in \mathbf{Z}$, so that $\beta_{j} \rho_{j}\left(g_{j}\right) \beta_{j}^{-1}(z)=z+n_{j}+m_{j} \tau_{j}$. Since $\left\{\beta_{j} \rho_{j}\left(g_{j}\right) \beta_{j}^{-1}\right\}$ converges to a Möbius transformation $\gamma$, it is easy to see that both $\left\{n_{j}\right\}$ and $\left\{m_{j}\right\}$ must be eventually constant, and so $\gamma \in \rho(G)$.

Lemma 3.6

Step 2: In this step, we establish Theorem 3.1 in the case in which the algebraic limit $\rho(G)$ has connected limit set. As we have already shown that Theorem 3.1 holds for elementary groups, generalized web groups, and degenerate groups without accidental parabolics, it will suffice to show that it is preserved by Klein-Maskit combination along a parabolic cyclic subgroup.

In order to cleanly handle comparisons of cusp regions of the limit of a convergent sequence and of the approximates, we use the fact that we are working with type-preserving sequences to make a convenient normalization. Let $\left\{\rho_{j}\right\} \subset \mathcal{D}(G)$ be a type-preserving sequence converging 
algebraically to $\rho$, and let $\rho(h)$ be a primitive parabolic element of $\rho(G)$. Since $\left\{\rho_{j}(h)\right\}$ is a sequence of parabolic Möbius transformations converging to $\rho(h)$, there exists a sequence $\left\{\beta_{j}\right\}$ of Möbius transformations converging to the identity so that $\beta_{j} \rho_{j}(h) \beta_{j}^{-1}(z)=\rho(h)$ for all $j$. Since $\left\{\beta_{j}\right\}$ converges to the identity, the modified sequence $\left\{\beta_{j} \rho_{j} \beta_{j}^{-1}\right\}$ has the same algebraic limit as the original sequence $\left\{\rho_{j}\right\}$, namely $\rho$, and the geometric limit of $\left\{\beta_{j} \rho_{j}(G) \beta_{j}^{-1}\right\}$ is equal to the geometric limit of the original sequence $\left\{\rho_{j}(G)\right\}$, if the geometric limit exists. Hence, we may replace the original sequence with the modified sequence without effect. We refer to this process as normalizing the sequence about $\rho(h)$.

We now wish to describe what we mean by cusp regions persisting in the approximates. Let $\left\{\rho_{j}\right\} \subset \mathcal{D}(G)$ be a type-preserving sequence converging to $\rho$ and let $D \subset \Omega(\rho(G))$ be a cusp region for $\rho(h)$. Say that $D$ persists in the approximates if, whenever $\left\{\rho_{j}\right\}$ is normalized about $\rho(h)$ to obtain a new sequence $\left\{\rho_{j}^{\prime}\right\}, D$ is a cusp region for $\rho_{j}^{\prime}(h)$ for all sufficiently large $j$.

In order to show that the conclusions of Theorem 3.1 are preserved under the application of the Klein-Maskit combination theorems, we need to carry along the information about the persistence of cusp regions. We begin by showing in Proposition 3.7 that cusp regions persist in the approximates for degenerate groups without accidental parabolic elements and for generalized web groups. In Proposition 3.9, we show that if $\rho(G)$ is obtained from $\rho\left(G_{1}\right)$ and $\rho\left(G_{2}\right)$ by Klein-Maskit combination along an accidental parabolic element, and if $\left\{\Lambda\left(\rho_{j}\left(G_{m}\right)\right\}\right.$ converges to $\Lambda\left(\rho\left(G_{m}\right)\right.$ and all cusp regions of $\rho\left(G_{m}\right)$ persist in the approximates for both $m$, then $\left\{\Lambda\left(\rho_{j}(G)\right)\right\}$ converges to $\Lambda(\rho(G)),\left\{\rho_{j}\right\}$ converges strongly to $\rho$, and all cusp regions of $\rho(G)$ persist in the approximates. Combining these two propositions, we construct an inductive proof of Theorem 3.1 in the case that $\rho(G)$ has connected limit set.

Proposition 3.7 Let $G$ be a finitely generated, torsion-free group, and let $\left\{\rho_{j}\right\} \subset \mathcal{D}(G)$ be a typepreserving sequence converging algebraically to $\rho \in \mathcal{D}(G)$. Suppose that $\rho(G)$ is either degenerate without accidental parabolics, generalized web, or elementary. Then, cusp regions persist in the approximates.

Proof of Proposition 3.7: We begin by noting that we have already shown that $\left\{\rho_{j}\right\}$ converges strongly to $\rho$ and that $\left\{\Lambda\left(\rho_{j}(G)\right)\right\}$ converges to $\Lambda(\rho(G))$. Let $D$ be a cusp region for the primitive parabolic $\rho(h)$. We assume that the sequence $\left\{\rho_{j}\right\}$ is normalized about $\rho(h)$. In the case that $\rho(G)$ is elementary, there is nothing to prove. Hence, we may assume that $\rho(G)$ is either degenerate without accidental parabolics or generalized web.

Let $c=\partial D \cap \Omega(\rho(G))$. The first key observation is that $c$ lies in $\Omega\left(\rho_{j}\left(G_{m}\right)\right)$ and is precisely invariant under $\left\langle\rho_{j}(h)\right\rangle$ in $\rho_{j}(G)$ for sufficiently large $j$. As this argument is used later, we state it as a lemma. 
Lemma 3.8 Let $G$ be a finitely generated, torsion-free, non-abelian group, and let $\left\{\rho_{j}\right\} \subset \mathcal{D}(G)$ be a type-preserving sequence converging strongly to $\rho$ such that $\left\{\Lambda\left(\rho_{j}(G)\right)\right\}$ converges to $\Lambda(\rho(G))$. Suppose that $D$ is a cusp region for $\rho(G)$ associated to the primitive parabolic element $\rho(h)$, that $\left\{\rho_{j}\right\}$ is normalized about $\rho(h)$, and set $c=\partial D \cap \Omega(\rho(G))$. Then, for sufficiently large $j$, c lies in $\Omega\left(\rho_{j}(G)\right)$ and is precisely invariant under $\left\langle\rho_{j}(h)\right\rangle$ in $\rho_{j}(G)$.

Proof of 3.8: We may assume that $\rho(h)(z)=z+1$. Choose a point $z$ on $c$, and let $c_{f}$ be the closed arc in $c$ joining $z$ to $z+1$, so that the interior of $c_{f}$ is a fundamental domain for the action of $\langle\rho(h)\rangle$ on $c$. Since $c_{f}$ is compact and since $\left\{\Lambda\left(\rho_{j}(G)\right)\right\}$ converges to $\Lambda(\rho(G))$, we see that $c_{f}$ lies in $\Omega\left(\rho_{j}(G)\right)$ for sufficiently large $j$, and hence that $c$ lies in $\Omega\left(\rho_{j}(G)\right)$ for sufficiently large $j$.

In order to show that $c$ is precisely invariant under $\left\langle\rho_{j}(h)\right\rangle$ in $\rho_{j}(G)$, we need to make use of a standard convergence property for Möbius transformations, which we describe here. Let $K$ be a compact set in $\overline{\mathbf{C}}$ and let $\left\{M_{j}\right\}$ be a sequence of distinct Möbius transformations so that $K \cap M_{j}(K)$ is non-empty for all $j$. Then, there exists a subsequence of $\left\{M_{j}\right\}$, again called $\left\{M_{j}\right\}$, so that either there exists a sequence of points $\left\{x_{j} \in \operatorname{fix}\left(M_{j}\right)\right\}$ converging to a point in $K$ or $\left\{M_{j}\right\}$ converges to a Möbius transformation (see Marden [19] or Gehring and Martin [13].)

If $c$ is not precisely invariant under $\left\langle\rho_{j}(h)\right\rangle$ in $\rho_{j}(G)$ for all sufficiently large $j$, there exists a sequence $\left\{g_{j}\right\}$ of elements of $G-\langle h\rangle$ so that $c \cap \rho_{j}\left(g_{j}\right)(c)$ is non-empty for infinitely many $j$. We may pre- and post-multiply each $g_{j}$ by appropriate powers of $h$ to produce a sequence $\left\{g_{j}^{\prime}\right\}$ of elements of $G-\langle h\rangle$ so that $c_{f} \cap \rho_{j}\left(g_{j}^{\prime}\right)\left(c_{f}\right)$ is non-empty for infinitely many $j$. The convergence property for Möbius transformations implies that there exists a subsequence of $\left\{\rho_{j}\left(g_{j}^{\prime}\right)\right\}$, again called $\left\{\rho_{j}\left(g_{j}^{\prime}\right)\right\}$, for which either there exist points $\left\{x_{j} \in \Lambda\left(\rho_{j}(G)\right)\right\}$ converging to a point in $c_{f}$, or $\left\{\rho_{j}\left(g_{j}^{\prime}\right)\right\}$ converges to a Möbius transformation $\gamma$. In the latter case, note that $c_{f} \cap \gamma\left(c_{f}\right)$ is necessarily non-empty.

The former case cannot occur, as $\left\{\Lambda\left(\rho_{j}(G)\right)\right\}$ converges to $\Lambda(\rho(G))$, which is disjoint from $c_{f}$. The latter case cannot occur, as the precise invariance of $c$ under $\langle\rho(h)\rangle$ in $\rho(G)$ and the strong convergence of $\left\{\rho_{j}\right\}$ to $\rho$ together imply that $\gamma=\rho\left(h^{n}\right)$ for some $n \neq 0$; Lemma 3.6 of Jørgensen and Marden [15] then implies that $g_{j}^{\prime}=h^{n}$ for all sufficiently large $j$, contradicting our assumption that $g_{j}^{\prime}$ does not lie in $\langle h\rangle$.

We now show that $D$ persists in the approximates. Suppose first that $\rho(G)$ is a degenerate group without accidental parabolics. As each isomorphism $\rho_{j} \circ \rho^{-1}: \rho(G) \rightarrow \rho_{j}(G)$ is type-preserving and $\Omega\left(\rho_{j}(G)\right.$ ) is non-empty for all large enough $j$, Theorem 6 of Maskit [21] gives that, for all large enough $j, \rho_{j}(G)$ does not contain accidental parabolic elements. However, since $c$ is precisely 
invariant under $\left\langle\rho_{j}(h)\right\rangle$ in $\rho_{j}(G)$ for all sufficiently large $j$, by Lemma 3.8, we see that either $D$ is a cusp region for $\rho_{j}(h)$ in $\rho_{j}(G)$, or $C=c \cup \operatorname{fix}(\rho(h))$ separates $\Lambda\left(\rho_{j}(G)\right)$, in which case $\rho_{j}(h)$ is an accidental parabolic element of $\rho_{j}(G)$. As the latter case cannot occur, it must be that $D$ is a cusp region for $\rho_{j}(h)$ in $\rho_{j}(G)$ for all sufficiently large $j$.

Suppose now that $\rho(G)$ is a generalized web group, let $\Delta$ be the component of $\Omega(\rho(G))$ containing $D$, and let $\Phi=\operatorname{st}_{\rho(G)}(\Delta)$. By Lemma 3.8, we know that $c$ lies in $\Omega\left(\rho_{j}(G)\right)$ and is precisely invariant under $\left\langle\rho_{j}(h)\right\rangle$ for all sufficiently large $j$. By Corollary 3.5, $\Phi_{j}=\rho_{j} \circ \rho^{-1}(\Phi)$ is a quasifuchsian component subgroup of $\rho_{j}(G)$, stabilizing the component $\Delta_{j}$ of $\rho_{j}(G)$. Since $\left\{\Lambda\left(\Phi_{j}\right)=\partial \Delta_{j}\right\}$ converges to $\Lambda(\Phi)=\partial \Delta$, we see that $c$ is contained in $\Delta_{j}$ for $j$ sufficiently large. Since quasifuchsian component subgroups cannot contain accidental parabolic elements (see Theorem 4 of Maskit [20]), the Jordan curve $C=\partial D$ cannot separate $\Lambda\left(\rho_{j}(G)\right)$, and so $D$ is a cusp region for $\rho_{j}(h)$ in $\rho_{j}(G)$ for all sufficiently large $j$.

Proposition 3.7

The following proposition shows that the conclusions of Theorem 3.1 are preserved by KleinMaskit combination along a cyclic parabolic subgroup.

Proposition 3.9 Let $G$ be a finitely generated, torsion-free, non-abelian group, and let $\left\{\rho_{j}\right\} \subset$ $\mathcal{D}(G)$ be a type-preserving sequence converging algebraically to $\rho$ with $\Omega(\rho(G))$ non-empty. Suppose that $\Theta=\langle\rho(h)\rangle$ is an accidental parabolic subgroup of $\rho(G)$, and that $\rho\left(G_{1}\right)$ and $\rho\left(G_{2}\right)$ are the factor subgroups of the Klein-Maskit decomposition of $\rho(G)$ along $\Theta$. If $\left\{\Lambda\left(\rho_{j}\left(G_{m}\right)\right)\right\}$ converges to $\Lambda\left(\rho\left(G_{m}\right)\right)$ for both $m$ and if all cusp regions for $\rho\left(G_{m}\right)$ persist in the approximates, then $\left\{\rho_{j}\right\}$ converges strongly to $\rho,\left\{\Lambda\left(\rho_{j}(G)\right)\right\}$ converges to $\Lambda(\rho(G))$, and all cusp regions for $\rho(G)$ persist in the approximates.

Proof of Proposition 3.9: We begin by showing that $\left\{\Lambda\left(\rho_{j}(G)\right)\right\}$ converges to $\Lambda(\rho(G))$. Proposition 2.2 then implies the strong convergence of $\left\{\rho_{j}\right\}$ to $\rho$. In order to show that the limit sets converge, we first show that the approximates are also Klein-Maskit combinations along (the same) cyclic parabolic subgroup. We use this to construct explicit fundamental domains for the approximates which can be used to show that the limit sets converge. We then show that the cusp regions for $\rho(G)$ persist in the approximates. The details for the two Klein-Maskit combinations are similar; we give full details for the case of a Klein-Maskit combination of type I, and briefly sketch the argument for a Klein-Maskit combination of type II at the end of the proof.

If $\left\{\Lambda\left(\rho_{j}\right)\right\}$ does not converge to $\Lambda(\rho(G))$, we may pass to a subsequence so that $\left\{\Lambda\left(\rho_{j}(G)\right)\right\}$ converges to a closed set $\widehat{\Lambda}$ containing $\Lambda(\rho(G))$ as a proper subset. Without loss of generality, we may assume that $\left\{\rho_{j}\right\}$ is normalized about $\rho(h)$ and that $\rho(h)(z)=z+1$. Let $c$ be the axis of 
$\rho(h)$ and let $C=c \cup\{\infty\}$ be the full axis of $\rho(h)$. Let $E_{1}$ and $E_{2}$ be the two closed discs in $\overline{\mathbf{C}}$ determined by $C$, labeled so that $E_{m}$ is a cusp region for $\rho(h)$ in $\rho\left(G_{m}\right)$.

Since cusp regions persist in the approximates for both $\rho\left(G_{m}\right)$, we have that $E_{m}$ is a cusp region for $\rho_{j}(h)$ in $\rho_{j}\left(G_{m}\right)$ for both $m$ and for all sufficiently large $j$. Therefore, $\rho_{j}(G)$ is formed from $\rho_{j}\left(G_{1}\right)$ and $\rho_{j}\left(G_{2}\right)$ by a Klein-Maskit combination of type I along $C$ for all sufficiently large $j$.

Choose a point $z$ on $c$ and consider the open vertical band $V$ in $\mathbf{C}$ whose two bounding lines pass through $z$ and $z+1$. For both $m$, we can choose a fundamental domain $F^{m}$ for $\rho\left(G_{m}\right)$ which lies in $V$ and contains $V \cap E_{m}$. Since $\rho(G)$ is formed from $\rho\left(G_{1}\right)$ and $\rho\left(G_{2}\right)$ by Klein-Maskit combination of type I along $C$, Theorem 2.4 assures us that $F=F^{1} \cap F^{2}$ is a fundamental domain for $\rho(G)$. Choose a point $x \in \widehat{\Lambda}-\Lambda(\rho(G))$ which lies in the interior of $F$ (noting that one may need to alter the choice of $F$ to guarantee that $x$ lies in $F$ rather than on its boundary). Let $K$ be a compact set which contains an open neighborhood of $x$ and which is contained entirely in the interior of $F$. Since $K$ lies in $F$, it is precisely invariant under the identity in $\rho(G)$.

By construction, $K$ lies in the strip $V$. Combining the convergence property for Möbius transformations (see the proof of Lemma 3.8) with the assumptions that $\left\{\rho_{j}\left(G_{m}\right)\right\}$ converges to $\rho\left(G_{m}\right)$ and $\left\{\Lambda\left(\rho_{j}\left(G_{m}\right)\right)\right\}$ converges to $\Lambda\left(\rho\left(G_{m}\right)\right)$, we see that $K$ lies in $\Omega\left(\rho_{j}\left(G_{m}\right)\right)$ and is precisely invariant under the identity in $\rho_{j}\left(G_{m}\right)$ for sufficiently large $j$. Moreover, since $E_{m}$ is precisely invariant under $\left\langle\rho_{j}(h)\right\rangle$ in $\rho_{j}\left(G_{m}\right)$ for sufficiently large $j$, we may construct a fundamental domain $F_{j}^{m}$ for $\rho_{j}\left(G_{m}\right)$ containing both $V \cap E_{m}$ and $K$ for sufficiently large $j$; in particular, $F_{j}^{1} \cap c=F_{j}^{2} \cap c$. Since $\rho_{j}(G)$ is formed from $\rho_{j}\left(G_{1}\right)$ and $\rho_{j}\left(G_{2}\right)$ by Klein-Maskit combination of type I along $C$, Theorem 2.4 implies that $F_{j}=F_{j}^{1} \cap F_{j}^{2}$ is then a fundamental domain for $\rho_{j}(G)$ for sufficiently large $j$.

Since $\left\{\Lambda\left(\rho_{j}(G)\right)\right\}$ converges to $\widehat{\Lambda}$, there exists a sequence of points $\left\{x_{j} \in \Lambda\left(\rho_{j}(G)\right)\right\}$ converging to $x$. This implies that $x_{j} \in K \subset F_{j} \subset \Omega\left(\rho_{j}(G)\right)$ for sufficiently large $j$, a contradiction. Hence, $\left\{\Lambda\left(\rho_{j}(G)\right)\right\}$ converges to $\Lambda(\rho(G))$, and so $\left\{\rho_{j}\right\}$ converges strongly to $\rho$.

In order to complete the proof in the case of a Klein-Maskit combination of type I, it remains only to argue that cusp regions for $\rho(G)$ persist in the approximates. Let $D^{\prime}$ be a cusp region for a primitive parabolic element $\rho(g)$ of $\rho(G)$. After suitably normalizing, we first use Klein-Maskit Combination Theorem 2.4 and our assumption that cusps persist in the approximates of the factors to show that a cusp region $D^{\prime \prime}$ contained in $D^{\prime}$ (and missing all translates of one of the cusp regions we are combining along) does persist in the approximates. We then use the convergence property and the fact that limit sets converge to show that $D^{\prime}$ itself must persist in the approximates.

Let $\left\{\beta_{j}\right\}$ be a sequence of Möbius transformations converging to the identity so that $\beta_{j} \rho_{j}(g) \beta_{j}^{-1}=$ $\rho(g)$ for all $j$. Set $\rho_{j}^{\prime}=\beta_{j} \circ \rho_{j} \circ \beta_{j}^{-1}$, and let $c_{j}=\beta_{j}(c)$ and $E_{m}^{j}=\beta_{j}\left(E_{m}\right)$ for all $j$ and for both $m$. Notice that, for all large enough $j, E_{m}^{j}$ is a cusp region for $\rho_{j}^{\prime}(h)$ in $\rho_{j}^{\prime}\left(G_{m}\right)$ and $\rho_{j}^{\prime}(G)$ is a Klein-Maskit combination of type I of $\rho_{j}^{\prime}\left(G_{1}\right)$ and $\rho_{j}^{\prime}\left(G_{2}\right)$ along $\left\langle\rho_{j}^{\prime}(h)\right\rangle$ with associated axis $c_{j}$. 
Moreover, $\left\{\rho_{j}^{\prime}\right\}$ converges strongly to $\rho$ and $\left\{\Lambda\left(\rho_{j}^{\prime}(G)\right)\right\}$ converges to $\Lambda(\rho(G))$.

Let $\xi$ be the fixed point of $\rho(g)$, and let $d^{\prime}=\partial D^{\prime}-\{\xi\}$. Lemma 3.8 implies that $d^{\prime}$ is precisely invariant under $\left\langle\rho_{j}(g)\right\rangle$ in $\rho_{j}(G)$ for sufficiently large $j$. By Theorem 2.4, every cusp region for $\rho(G)$ is a cusp region for either $\rho\left(G_{1}\right)$ or $\rho\left(G_{2}\right)$, and so we may assume that $D^{\prime}$ is a cusp region for $\rho\left(G_{1}\right)$. Since we have assumed that cusp regions for $\rho_{j}\left(G_{1}\right)$ persist in the approximates, we see that $D^{\prime}$ is a cusp region for $\rho_{j}^{\prime}\left(G_{1}\right)$ for all sufficiently large $j$. Moreover, since $E_{1}$ is not a cusp region for $\rho(G)$, we see that $D^{\prime}$ and $E_{1}$ are inequivalent cusp regions for $\rho\left(G_{1}\right)$, and hence that $D^{\prime}$ and $E_{j}^{1}$ are inequivalent cusp regions for $\rho_{j}\left(G_{1}\right)$ for all sufficiently large $j$.

Let $D^{\prime \prime} \subset D^{\prime}$ be a cusp region for $\rho(g)$ in $\rho\left(G_{1}\right)$ which does not intersect any $\rho\left(G_{1}\right)$-translate of $E_{1}$, and suppose that there exists a $\rho_{j}^{\prime}\left(G_{1}\right)$-translate of $E_{1}^{j}$ which intersects $D^{\prime \prime}$ for infinitely many $j$. Since $D^{\prime \prime}$ and $E_{1}^{j}$ are inequivalent for all sufficiently large $j, D^{\prime \prime}$ cannot contain or be contained in any translate of $E_{1}^{j}$. Hence, $d^{\prime \prime}=\partial D^{\prime \prime} \cap \Omega\left(\rho_{j}^{\prime}\left(G_{1}\right)\right)$ must intersect some $\rho_{j}^{\prime}\left(G_{1}\right)$-translate of $e^{\prime \prime}=\partial E_{1}^{j} \cap \Omega\left(\rho_{j}^{\prime}\left(G_{1}\right)\right)$ for infinitely many $j$. This, however, can be ruled out by the argument in the proof of Lemma 3.8. Hence, for all sufficiently large $j, D^{\prime \prime}$ does not intersect any $\rho_{j}^{\prime}\left(G_{1}\right)$-translate of $E_{1}^{j}$, and so Theorem 2.4 guarantees that $D^{\prime \prime}$ is a cusp region for $\rho_{j}^{\prime}(G)$ for sufficiently large $j$. That is, $D^{\prime \prime}$ persists in the approximates of $\rho(G)$.

Suppose that $D^{\prime}$ itself does not persist in the approximates of $\rho(G)$. Then, for infinitely many $j$, there exists $g_{j} \in G-\langle g\rangle$ such that $\rho_{j}^{\prime}\left(g_{j}\right)\left(D^{\prime}\right)$ intersects $D^{\prime}$. Since $d^{\prime}$ is precisely invariant under $\left\langle\rho_{j}(g)\right\rangle$ in $\rho_{j}(G)$ for sufficiently large $j$, this implies that $\rho_{j}\left(g_{j}\right)(\xi)$ is contained in the interior of $D^{\prime}$ for infinitely many $j$. However, the argument in the proof of Lemma 3.8 shows that $D^{\prime}-D^{\prime \prime} \subset \Omega\left(\rho_{j}(G)\right)$ for all sufficiently large $j$. (The key point is that there is a compact fundamental domain for the action of $\langle\rho(g)\rangle$ on $D^{\prime}-\operatorname{int}\left(D^{\prime \prime}\right)$, where $\operatorname{int}\left(D^{\prime \prime}\right)$ is the interior of $D^{\prime \prime}$.) Since $D^{\prime \prime}$ is a cusp region for $\rho_{j}(G)$ for all sufficiently large $j$, we see that the interior of $D^{\prime}$ must be contained in $\Omega\left(\rho_{j}(G)\right)$ for all sufficiently large $j$, a contradiction. Hence, $D^{\prime}$ is a cusp region for $\rho_{j}^{\prime}(G)$ for all sufficiently large $j$, and we have completed the proof of Proposition 3.9 in the case that $\rho(G)$ is formed from $\rho\left(G_{1}\right)$ and $\rho\left(G_{2}\right)$ by a Klein-Maskit combination of type I.

The proof in the case of Klein-Maskit combination of type II is quite similar. Suppose that the factor subgroups of the decomposition are $\rho(H)$ and $\langle\rho(g)\rangle$. Let $C_{1}, C_{2}, E_{1}, E_{2}, \theta_{1}=\rho\left(h_{1}\right)$ and $\theta_{2}=\rho\left(h_{2}\right)$ be as in the statement of the Klein-Maskit combination theorem of type II. We may assume that our sequence is normalized about $\rho\left(h_{1}\right)$. Let $c_{m}=C_{m} \cap \Omega(H)$. Let $c_{1}^{j}=c_{1}, E_{1}^{j}=E_{1}$, $c_{2}^{j}=\rho_{j}(g)\left(c_{1}\right)$ and $E_{2}^{j}=\rho_{j}(g)\left(E_{2}\right)$. By assumption, for sufficiently large $j, E_{m}^{j}$ is a cusp region for $\rho_{j}\left(h_{m}\right)$ in $\rho_{j}(H)$. As before, we see that $\rho_{j}(G)$ is a Klein-Maskit combination of type II with factor subgroups $\rho_{j}(H)$ and $\left\langle\rho_{j}(g)\right\rangle$, for sufficiently large $j$, and that $\left\{\Lambda\left(\rho_{j}(G)\right)\right\}$ converges to $\Lambda(\rho(G))$. This again implies that $\left\{\rho_{j}\right\}$ converges strongly to $\rho$. The proof that cusp regions persist in the approximates is also much as above. 
Proposition 3.9

We are now prepared to establish Theorem 3.1 in the case when $\rho(G)$ has connected limit set. We suppose that $\left\{\rho_{j}\right\} \subset \mathcal{D}(G)$ is a type-preserving sequence converging algebraically to $\rho$, that $\Omega(\rho(G))$ is non-empty, and that $\Lambda(\rho(G))$ is connected. Let $\left\{\Gamma_{1}, \ldots, \Gamma_{k}, \Gamma_{k+1}, \ldots, \Gamma_{l}\right\}$ be the Abikoff-Maskit decomposition of $\rho(G)$ as described in Section 2.3, so that each $\Gamma_{i}$ is either degenerate without accidental parabolics, generalized web or cyclic. Let $H_{i}=\rho^{-1}\left(\Gamma_{i}\right)$. By the results of Step 1, each of the restricted sequences $\left\{\left.\rho_{j}\right|_{H_{i}}\right\}$ converges strongly to $\left.\rho\right|_{H_{i}}$ and $\left\{\Lambda\left(\rho_{j}\left(H_{i}\right)\right)\right\}$ converges to $\Lambda\left(\rho\left(H_{i}\right)\right)$. Proposition 3.7 implies that all cusp regions of $\rho\left(H_{i}\right)$ persist in the approximates. We set $\Gamma^{1}=\Gamma_{1}$, and recall that $\Gamma^{i}$ is formed from $\Gamma^{i-1}$ and $\Gamma_{i}$ by Klein-Maskit combination along a parabolic cyclic subgroup, of type I for $1 \leq i \leq k$ and of type II for $k<i$, and $\rho(G)=\Gamma^{l}$. Let $H^{i}=\rho^{-1}\left(\Gamma^{i}\right)$. Applying Proposition 3.9 inductively shows, for all $i$, that $\left\{\left.\rho_{j}\right|_{H^{i}}\right\}$ converges strongly to $\left.\rho\right|_{H^{i}}$, that $\left\{\Lambda\left(\rho_{j}\left(H^{i}\right)\right)\right\}$ converges to $\Lambda\left(\rho\left(H^{i}\right)\right)$, and that all cusp regions of $\rho\left(H^{i}\right)$ persist in the approximates. This completes the proof in the case that $\rho(G)$ has connected limit set.

Step 3: We conclude the proof of the theorem by considering the case in which $\rho(G)$ has nonconnected limit set. In this case we may decompose $\rho(G)$ into a finite collection of subgroups, each of which either has connected limit set or is elementary, so that $\rho(G)$ is built up from the subgroups by repeatedly applying the operation of Klein combination. One may then complete the proof of the theorem by applying the following result from [4] finitely many times.

Proposition 3.10 (Proposition 10.2 in [4]) Let $G$ be a finitely generated, torsion-free group, and let $\left\{\rho_{j}\right\} \subset \mathcal{D}(G)$ be a type-preserving sequence converging algebraically to $\rho \in \mathcal{D}(G)$. Suppose that $\Omega(\rho(G))$ is non-empty and $\rho(G)$ is obtained from $\rho\left(G_{1}\right)$ and $\rho\left(G_{2}\right)$ by Klein combination. If $\left\{\Lambda\left(\rho_{j}\left(G_{m}\right)\right)\right\}$ converges to $\Lambda\left(\rho\left(G_{m}\right)\right)$ for both $m$, then $\left\{\rho_{j}\right\}$ converges strongly to $\rho$ and $\left\{\Lambda\left(\rho_{j}(G)\right)\right\}$ converges to $\Lambda(\rho(G))$.

Theorem 3.1

\section{Generalizations}

One may use Selberg's lemma (see Selberg [30]) and results of Jørgensen and Marden to remove the assumption that $G$ is torsion-free in the statement of Theorem 3.1.

Theorem 4.1 Let $G$ be a finitely generated group and let $\left\{\rho_{j}\right\} \subset \mathcal{D}(G)$ be a type-preserving sequence converging algebraically to $\rho \in \mathcal{D}(G)$. If $\Omega(\rho(G))$ is non-empty, then $\left\{\rho_{j}\right\}$ converges strongly to $\rho$ and $\left\{\Lambda\left(\rho_{j}(G)\right)\right\}$ converges to $\Lambda(\rho(G))$. 
Proof of 4.1: Selberg's lemma guarantees that there exists a finite index, torsion-free subgroup $H$ of $G$. Theorem 3.1 implies that $\left\{\left.\rho_{j}\right|_{H}\right\}$ converges strongly to $\left.\rho\right|_{H}$ and that $\left\{\Lambda\left(\rho_{j}(H)\right)\right\}$ converges to $\Lambda(\rho(H))$. Since $\Lambda\left(\rho_{j}(G)\right)=\Lambda\left(\rho_{j}(H)\right)$ for all $j$ and $\Lambda(\rho(G))=\Lambda(\rho(H))$, we see that $\left\{\Lambda\left(\rho_{j}(G)\right)\right\}$ converges to $\Lambda(\rho(G))$. If $G$ is not virtually abelian, then Proposition 2.2 implies that $\left\{\rho_{j}\right\}$ converges strongly to $\rho$. If $G$ is virtually abelian, we may prove that $\left\{\rho_{j}\right\}$ converges strongly to $\rho$ by extending the arguments given in the proof of Lemma 3.6.

We can similarly generalize Corollary 3.3 to obtain:

Corollary 4.2 Let $G$ be a finitely generated group and let $\left\{\rho_{j}\right\} \subset \mathcal{D}(G)$ be a type-preserving sequence converging algebraically to $\rho$. If $G$ contains a finite index, torsion-free subgroup which is not a (non-trivial) free product of (orientable) surface groups and infinite cyclic groups, then $\left\{\rho_{j}\right\}$ converges strongly to $\rho$ and $\left\{\Lambda\left(\rho_{j}(G)\right)\right\}$ converges to $\Lambda(\rho(G))$.

\section{References}

[1] W. Abikoff and B. Maskit, 'Geometric decompositions of Kleinian group', Amer. J. Math. 99 (1977), 687-697.

[2] L. V. Ahlfors, 'Finitely generated Kleinian groups', Amer. J. Math. 86 (1964), 413-29.

[3] J. W. Anderson and R. D. Canary, 'Algebraic limits of Kleinian groups which rearrange the pages of a book', Invent. Math., 126 (1996), 205-214.

[4] J. W. Anderson and R. D. Canary, 'Cores of hyperbolic 3-manifolds and limits of Kleinian groups', Amer. J. Math. 118 (1996), 745-779.

[5] J. W. Anderson, R. D. Canary, M. Culler, and P. Shalen, 'Free Kleinian groups and volumes of hyperbolic 3-manifolds', J. Differential Geom. 44 (1996), 738-782.

[6] R. Benedetti and C. Petronio, Lectures on Hyperbolic Geometry, Springer-Verlag Universitext, 1992.

[7] F. Bonahon, 'Bouts des variétés hyperboliques de dimension 3', Annals Math. 124 (1986), 71-158.

[8] R. D. Canary, 'Ends of hyperbolic 3-manifolds', J.A.M.S. 6 (1993), 1-35.

[9] R. D. Canary, 'A covering theorem for hyperbolic 3-manifolds and its applications', Topology, 35 (1996), 751778.

[10] R. D. Canary, D. B. A. Epstein, and P. Green, 'Notes on notes of Thurston', in Analytical and Geometrical Aspects of Hyperbolic Spaces, Cambridge University Press, 1987, 3-92.

[11] A. Douady and C.J. Earle, 'Conformally natural extensions of homeomorphisms of the circle', Acta Math. 157 (1986) 23-48. 
[12] D. B. A. Epstein and A. Marden, 'Convex hulls in hyperbolic spaces, a theorem of Sullivan, and measured pleated surfaces', in Analytical and Geometrical Aspects of Hyperbolic Spaces, Cambridge University Press, 1987, 113-253.

[13] F.W. Gehring and G.J. Martin, 'Discrete quasiconformal groups I', Proceedings L. M. S. 55 (1987), 331-358.

[14] T. Jørgensen, 'On discrete groups of Möbius transformations', Amer. J. Math. 98 (1976), 739-749.

[15] T. Jørgensen and A. Marden, 'Algebraic and geometric convergence of Kleinian groups', Math. Scand. 66 (1990), $47-72$.

[16] S. Kerckhoff and W. P. Thurston, 'Non-continuity of the action of the mapping class group at Bers' boundary of Teichmuller space', Invent. Math. 100 (1990), 25-47.

[17] R. Kulkarni and P. Shalen, 'On Ahlfors' finiteness theorem', Adv. in Math. 76 (1989), 155-169.

[18] A. Marden, 'The geometry of finitely generated Kleinian groups', Annals Math. 99 (1974), 383-462.

[19] A. Marden, 'Geometrically finite Kleinian groups and their deformation spaces', in Discrete Groups and Automorphic Functions, ed. by W. J. Harvey, Academic Press, New York, 1977, 259-293.

[20] B. Maskit, 'On boundaries of Teichmüller spaces and on Kleinian groups II', Annals Math. 91 (1970), 607-639.

[21] B. Maskit, 'On the classification of Kleinian groups: I - Koebe groups', Acta Math. 135 (1975), 249-270.

[22] B. Maskit, Kleinian groups, Springer-Verlag, 1988.

[23] D. McCullough, 'Compact submanifolds of 3-manifolds with boundary', Quart. J. Math. Oxford 37 (1986), 299-307.

[24] K. Ohshika, 'Strong convergence of Kleinian groups and Caratheodory convergence of domains of discontinuity', Math. Proc. Camb. Phil. Soc. 112 (1992), 297-307.

[25] K. Ohshika, 'Geometric behaviour of Kleinian groups on boundaries for deformation spaces', Quart. J. Math. Oxford 43 (1992), 97-111.

[26] K. Ohshika, 'Kleinian groups which are limits of geometrically finite groups', preprint.

[27] K. Ohshika, 'Divergent sequences of Kleinian groups', preprint.

[28] H. M. Reimann, 'Invariant extensions of quasiconformal deformations', Ann. Acad. Sci. Fenn. 10 (1985), 477492.

[29] G. P. Scott, 'Compact submanifolds of 3-manifolds', Journal L. M. S. 7 (1973), 246-250.

[30] A. Selberg, 'On Discontinuous groups in higher dimensional symmetric spaces', in Colloquim Function Theory, Tata Institute, 1960.

[31] T. Soma, 'Function groups in Kleinian groups', Math. Annalen 292 (1992), 181-190.

[32] W. P. Thurston, The geometry and topology of 3-manifolds, lecture notes, Princeton University. 
[33] P. Tukia, 'Quasiconformal extensions of quasisymmetric mappings compatible with a Möbius group', Acta Math. 154 (1985), 153-193. 